\title{
Structural basis for the recognition of Asef by adenomatous polyposis coli
}

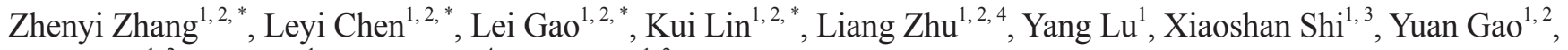
Jing Zhou ${ }^{1,2}$, Ping $\mathrm{Xu}^{1}$, Jian Zhang ${ }^{4}$, Geng $\mathrm{Wu}^{1,2}$

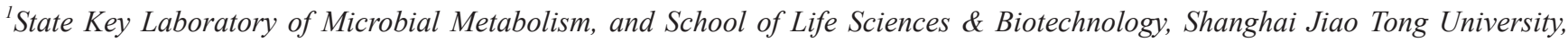
Shanghai 200240, China; ${ }^{2}$ Key Laboratory of MOE for Developmental Genetics and Neuropsychiatric Diseases, Shanghai Jiao Tong University, Shanghai 200240, China; ${ }^{3}$ Institute of Biochemistry and Cell Biology, Shanghai Institutes for Biological Sciences, Chinese Academy of Sciences, Shanghai 200031, China; ${ }^{4}$ Department of Pathophysiology, Key Laboratory of Cell Differentiation and Apoptosis of Chinese Ministry of Education, Shanghai Jiao-Tong University School of Medicine (SJTU-SM), Shanghai 200025, China

Adenomatous polyposis coli (APC) regulates cell-cell adhesion and cell migration through activating the APCstimulated guanine nucleotide-exchange factor (GEF; Asef), which is usually autoinhibited through the binding between its Src homology 3 (SH3) and Dbl homology (DH) domains. The APC-activated Asef stimulates the small GTPase Cdc42, which leads to decreased cell-cell adherence and enhanced cell migration. In colorectal cancers, truncated APC constitutively activates Asef and promotes cancer cell migration and angiogenesis. Here, we report crystal structures of the human APC/Asef complex. We find that the armadillo repeat domain of APC uses a highly conserved surface groove to recognize the APC-binding region (ABR) of Asef, conformation of which changes dramatically upon binding to APC. Key residues on APC and Asef for the complex formation were mutated and their importance was demonstrated by binding and activity assays. Structural superimposition of the APC/Asef complex with autoinhibited Asef suggests that the binding between APC and Asef might create a steric clash between AsefDH domain and APC, which possibly leads to a conformational change in Asef that stimulates its GEF activity. Our structures thus elucidate the molecular mechanism of Asef recognition by APC, as well as provide a potential target for pharmaceutical intervention against cancers.

Keywords: APC; Asef; cell adhesion and cell migration; cancer; armadillo repeat domain

Cell Research (2012) 22:372-386. doi:10.1038/cr.2011.119; published online 26 July 2011

\section{Introduction}

Mutations in the human tumor suppressor adenomatous polyposis coli $(A P C)$ gene are major causes of hereditary and sporadic colorectal cancers $[1,2]$. The human APC protein contains an oligomerization domain (OD), an armadillo repeat domain (ARM) consisting of seven armadillo repeats $[1,3]$, a region containing multiple $\beta$-catenin-

\footnotetext{
*These four authors contributed equally to this work. Correspondence: Geng Wu ${ }^{\mathrm{a}}$, Kui Lin ${ }^{\mathrm{b}}$, Jian Zhang ${ }^{\mathrm{c}}$

${ }^{a}$ E-mail: geng.wu@sjtu.edu.cn

bE-mail: klin@sjtu.edu.cn

${ }^{c}$ E-mail: jian.zhang@sjtu.edu.cn

Received 16 December 2010; revised 21 March 2011; accepted 4 May 2011; published online 26 July 2011
}

binding repeats and axin-binding repeats, and a basic domain that interacts with microtubules (Supplementary information, Figure S1A). A comparison of various APC orthologs and paralogs shows that the seven armadillo repeats and the region preceding the seven armadillo repeats (referred to as the PreARM region hereafter) are the most highly conserved regions in APC, with about $15 \%$ of their residues identical among all APC homologs (Supplementary information, Figure S2). Similar to the ARM domain of $\beta$-catenin [4-10], the ARM domain of APC provides a structural platform on which many interaction partners bind, including APC-stimulated guanine nucleotide exchange factor (GEF; Asef) [11], IQGAP1 [12], and KAP-3 [13].

APC is a major component in the Wnt signaling pathway and promotes the phosphorylation and ubiquitination 
of $\beta$-catenin $[14,15]$. In colorectal cancers, mutations in the $A P C$ gene usually occur as truncation mutations in its mutation cluster region, resulting in a truncated APC fragment with intact PreARM and ARM regions. The truncated APC is defective in the regulation of $\beta$-catenin phosphorylation and ubiquitination, and might instead function as an activator for Wnt signaling through promotion of Axin degradation [16].

Independent of its role in Wnt signaling, APC is also involved in the regulation of cell-cell adhesion and cell migration, partly through the recognition and activation of Asef. APC interacts via its ARM domain with the ABR region of Asef and its close homolog Asef2, which function as Dbl-family GEFs for the small Rho-like GTPase Cdc42 [11, 17-22]. In addition to the ABR region, Asef also contains an Src homology 3 (SH3) domain, a

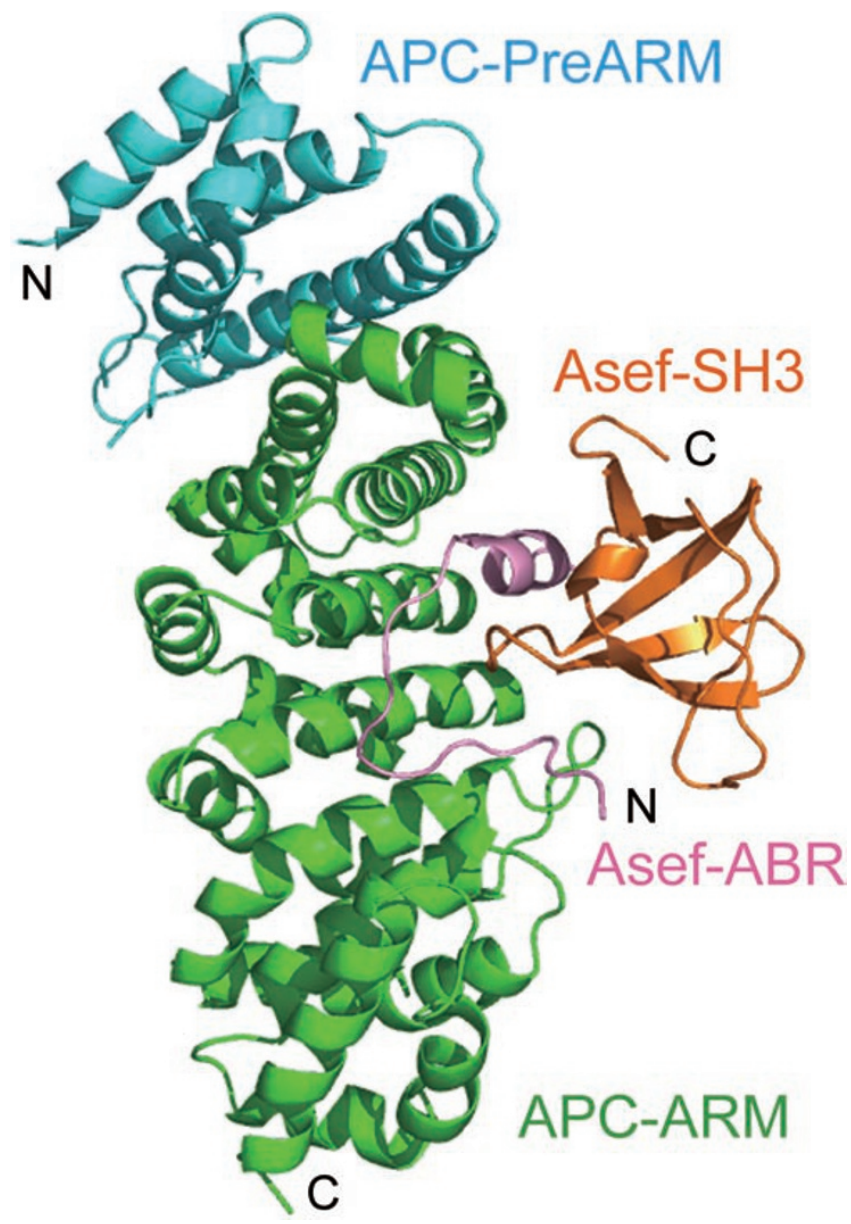

Figure 1 The crystal structure of the APC (residues 303-739, PreARM-ARM)/Asef (residues 170-271, ABR-SH3) complex. The PreARM and ARM portions of APC, and the ABR and SH3 domains of Asef are shown in cyan, green, pink, and orange, respectively.
Dbl homology (DH) domain, and a pleckstrin homology (PH) domain (Supplementary information, Figure S1B) $[11,23]$. The crystal structures of Asef by itself show that its $\mathrm{SH} 3$ domain interacts with its $\mathrm{DH}$ and $\mathrm{PH}$ domains, obstructs the binding of $\mathrm{Cdc} 42$ to the $\mathrm{DH}$ domain, and autoinhibits its GEF activity [22, 24]. On binding to APC, the autoinhibition of Asef is released and the GEF activity of Asef is stimulated. The activated Asef catalyzes the exchange of GDP for GTP in Cdc42, decreases cell-cell adhesion, and promotes cell migration [17, 2022]. In colorectal cancers, truncated APC constitutively activates Asef and $\mathrm{Cdc} 42$, which upregulate the expression of matrix metalloproteinase 9 (MMP9) via the c-Jun $\mathrm{N}$-terminal kinase (JNK) pathway, thus promotes cancercell migration and angiogenesis [25-27].

In this study, we determined the crystal structure of the PreARM-ARM domain of human APC in complex with the ABR-SH3 domains of human Asef, as well as the crystal structures of APC-ARM both by itself and in complex with Asef-ABR. Our structures reveal that APC-PreARM-ARM uses a highly conserved surface groove, formed by the $\mathrm{H} 3$ helices of armadillo repeats 1 to 4 and the $\mathrm{H} 1$ helix of armadillo repeat 3, to recognize Asef-ABR. Key residues on APC and Asef important for the complex formation were confirmed by binding and fluorescence-based GEF activity assays using sitedirected mutants. On binding to APC, the conformation of Asef-ABR changes dramatically. Structural superimposition of the APC/Asef complex with autoinhibited Asef reveals that the Asef-DH domain would collide with APC on APC/Asef binding, which might initiate the dissociation between Asef-DH and Asef-SH3 domains, and thus stimulate the Asef activity.

\section{Results}

Structure determination and the overall structure of the adenomatous polyposis coli-PreARM-ARM/Asef-ABRSH3 complex

To understand the structural basis of how APC recognizes Asef, we first determined the crystal structure of human APC (residues 407-751, APC-ARM) at $1.6 \AA$ resolution by single-wavelength anomalous dispersion (SAD) phasing of selenomethionyl proteins (Table 1). Using the structure of APC (407-751) as a searching model, we also determined the $2.3 \AA$ resolution structure of APC-ARM in complex with human Asef (residues 170-194, Asef-ABR; Supplementary information, Figure S3) and the $3.0 \AA$ resolution structure of APC (residues 303-739, APC-PreARM-ARM) in complex with Asef (residues 170-271, Asef-ABR-SH3; Figure 1) by molecular replacement (Table 1). The structure of APC-ARM 
Table 1 Data collection, phasing, and refinement statistics

\begin{tabular}{|c|c|c|c|}
\hline & $\begin{array}{l}\text { SeMet-APC } \\
(407-751)\end{array}$ & $\begin{array}{l}\text { APC (407-751)/Asef } \\
(170-194)\end{array}$ & $\begin{array}{l}\text { APC (303-739)/Asef } \\
(170-271)\end{array}$ \\
\hline \multicolumn{4}{|l|}{ Data collection } \\
\hline Wavelength & 0.97916 & 0.97939 & 1.00000 \\
\hline Unit-cell parameters $(\AA)$ & $\mathrm{a}=51.4, \mathrm{~b}=52.7, \mathrm{c}=63.5$ & $\mathrm{a}=147.6, \mathrm{~b}=92.1, \mathrm{c}=107.7$ & $\mathrm{a}=\mathrm{b}=163.2, \mathrm{c}=242.6$ \\
\hline Resolution range $(\AA)$ (outer shell) & $50.0-1.60(1.66-1.60)$ & $50.0-2.30(2.38-2.30)$ & $50.0-3.00(3.11-3.00)$ \\
\hline Completeness (\%) (outer shell) & $95.1(83.1)$ & $95.1(72.2)$ & $96.1(69.2)$ \\
\hline Redundancy (outer shell) & $1.9(1.3)$ & $7.1(4.9)$ & $5.6(4.3)$ \\
\hline Total observations/Unique reflections & $308,016 / 165,928$ & $432,532 / 60,878$ & $260,108 / 46,293$ \\
\hline Se sites found (expected) & $29(30)$ & & \\
\hline FOM & 0.49 & & \\
\hline \multicolumn{4}{|l|}{ Refinement } \\
\hline $\mathrm{R}$ factor $/ \mathrm{R}_{\text {free }}(\%)$ & $16.8 / 20.1$ & $20.8 / 25.7$ & $25.1 / 28.6$ \\
\hline Overall B-factor & 18.4 & 10.1 & 23.5 \\
\hline Rmsd bond lengths $(\AA)$ & 0.014 & 0.005 & 0.005 \\
\hline Rmsd bond angles $\left({ }^{\circ}\right)$ & 1.414 & 0.827 & 0.874 \\
\hline $\begin{array}{l}\text { Ramachandran plot (preferred, allowed, } \\
\text { disallowed, \% ) }\end{array}$ & $98.5,1.5,0$ & $98.7,1.3,0$ & $96.4,3.3,0.3$ \\
\hline
\end{tabular}

$R_{\text {merge }}=\Sigma_{h} \Sigma_{i}\left|I_{h, i}-I_{\mathrm{h}}\right| / \Sigma_{\mathrm{h}} \Sigma_{\mathrm{i}} I_{h, i}$ for the intensity $(I)$ of observation i of reflection $h$. Phasing power $=\mathbb{C} F_{i}^{\mathrm{TM}} / E$, where $\mathbb{C} F_{i}^{\mathrm{TM}}$ is the root-meansquare (rms) heavy atom structure factor and $E$ is the lack of closure error. $R$ factor $=\Sigma|| F_{\text {obs }}|-| F_{\text {calc }}|| \Sigma\left|F_{\text {obs }}\right|$, where $F_{\text {obs }}$ and $F_{\text {calc }}$ are the observed and calculated structure factors, respectively. $R_{\text {free }}=R$ factor calculated using $5 \%$ of the reflection data chosen randomly and omitted from the start of refinement. Rmsd, root-mean-square deviations from ideal geometry. Data for the highest resolution shell are shown in parentheses.

in complex with Asef-ABR is similar to that by itself, and they can be superimposed with a root-means-square distance (RMSD) of $0.835 \AA$ for aligned $\mathrm{C} \alpha$ atoms. The structures of the APC-PreARM-ARM/Asef-ABR-SH3 complex and the APC-ARM/Asef-ABR complex are also very similar to each other $(0.477 \AA$ RMSD for aligned $\mathrm{C} \alpha$ atoms). Therefore, we will only focus on the structure of the APC-PreARM-ARM/Asef-ABR-SH3 complex hereafter, unless especially mentioned otherwise.

In one asymmetric unit of the crystal structure, there are two heterodimers of the APC-PreARM-ARM/AsefABR-SH3 complex (Table 1). However, there is little intermolecular interaction between the two heterodimers. In addition, our gel filtration chromatography results showed that the complex was a heterodimer and not a heterotetramer in solution (data not shown), suggesting that further dimerization of the heterodimers in the asymmetric unit may result from crystal packing. Therefore, the structure of the APC-PreARM-ARM/Asef-ABR-SH3 complex is presented as a heterodimer below. The two heterodimers in the asymmetric unit are almost identical, with a $0.380 \AA$ RMSD for aligned $\mathrm{C} \alpha$ atoms. Thus, we will only discuss one of the heterodimers (chain A: APCPreARM-ARM, and chain L: Asef-ABR-SH3) as an example.

In our structure, APC-PreARM and APC-ARM fold together into a cylindrical superhelix, with Asef-ABR binding to the surface groove of the superhelix (Figure 1). The N-terminal portion of Asef-ABR (residues 170-185) forms an extended region, and the C-terminal portion of 
Asef-ABR (residues 186-192) folds into a single $\alpha$-helix. Both the N-terminal-extended region and the C-terminal $\alpha$-helix of Asef-ABR pack against the Asef-SH3 domain, which has little interaction with APC-PreARM-ARM.

\section{Structure of the PreARM-ARM domain of APC}

Similar to the ARM domain of $\beta$-catenin (Figure 2A) $[4,28]$, APC-PreARM-ARM forms a right-handed superhelix, with each armadillo repeat consisting of three $\alpha$-helices (H1, H2, and $\mathrm{H} 3$ ) packing extensively with its neighboring repeats (Figure 2B). Alignment of APC armadillo repeats shows that core residues of $\mathrm{H} 1, \mathrm{H} 2$, and $\mathrm{H} 3$ helices have consensus patterns similar to those of armadillo repeats of $\beta$-catenin [28] and plakophilin 1 (Figure 2C) [29]. The ARM domain of APC was previously regarded as consisting of seven armadillo repeats (residues 453-767) and forming a folding unit by itself $[1$, 3]. However, we find that the PreARM portion of APC (residues 326-442) packs extensively with APC-ARM and forms a single-folding unit together with it. APCPreARM is the second most highly conserved portion in APC (Supplementary information, Figure S2). It forms a helical structure consisting of five $\alpha$-helices (Figure 2D). The first four $\alpha$-helices fold into an armadillo repeat-like
A

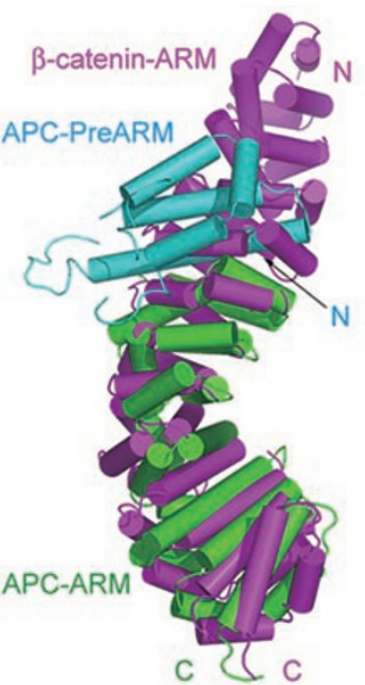

B

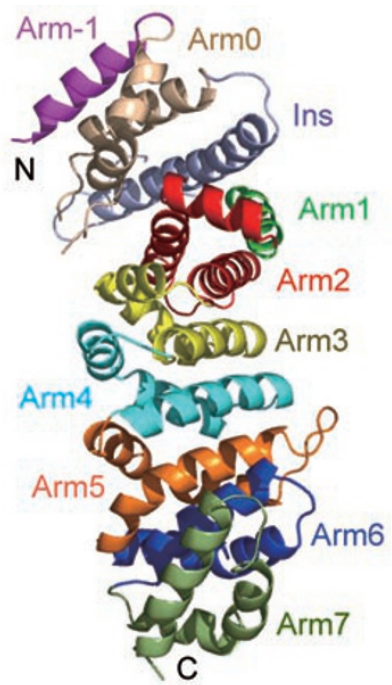

D

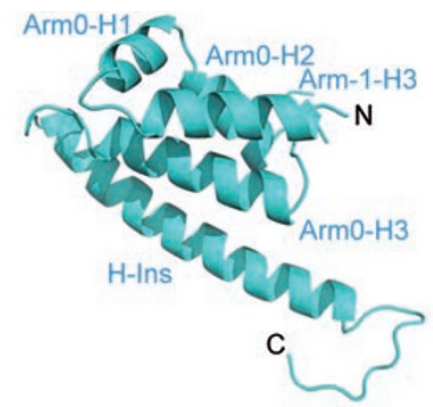

E

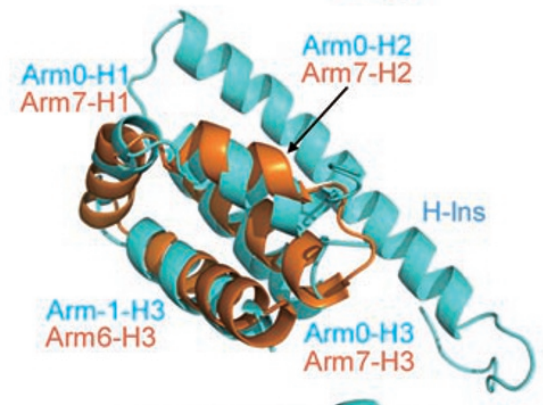

$\mathrm{F}$

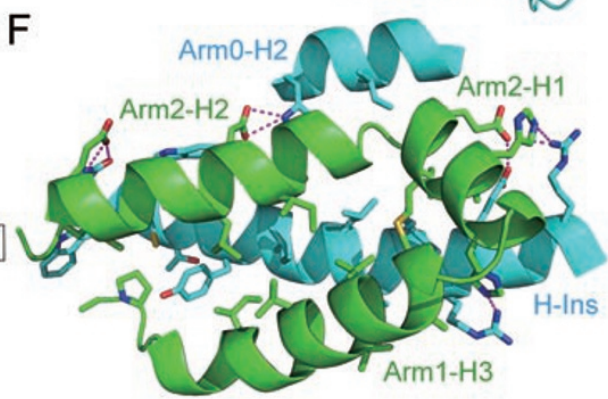

Figure 2 Structure of the PreARM-ARM domain of APC. (A) Structural comparison of the APC-PreARM-ARM domain with mouse $\beta$-catetin-ARM domain [27]. (B) Structure of the PreARM-ARM domain of APC. Each armadillo repeat and the long helix insertion (H-Ins) of APC-PreARM-ARM are shown in different colors. (C) Structure-based sequence alignment of human APC armadillo repeats. Residues forming helices $\mathrm{H} 1, \mathrm{H} 2$, and $\mathrm{H} 3$ are boxed with black lines. The long helix insertion ( $\mathrm{H}-\mathrm{Ins}$ ) of APC-PreARM is boxed with red lines. Consensus of APC armadillo repeats is shown below the sequences.,$+ \Phi$, and $x$ represent positively charged, hydrophobic, and any residue, respectively. (D) Structure of APC-PreARM. Secondary structure elements of APC-PreARM are labeled. (E) The structure of APC-PreARM (colored in cyan) is superimposed onto the structure of Arm6-H3 through Arm7-H3 of APC-ARM (colored in orange). Secondary structure elements are labeled. (F) Interface between APC-PreARM and APC-ARM. APC-PreARM is colored in cyan, while APC-ARM is colored in green. Secondary structure elements of APC-PreARM and APC-ARM that contribute to the interaction are indicated. Hydrogen bonds are represented by magenta dashed lines. 
structure, whereas the last $\alpha$-helix is much longer than the other ones (Figure 2E). Interestingly, we find that hydrophobic residues of the first four $\alpha$-helices of APCPreARM also conform to the consensus pattern of hydrophobic residues of APC armadillo repeats, corresponding to an $\mathrm{H} 3-\mathrm{H} 1-\mathrm{H} 2-\mathrm{H} 3$ sequence. As in APC-ARM, these hydrophobic residues of APC-PreARM interact extensively among each other. Therefore, we conclude that the entire APC-PreARM-ARM region forms one armadillo repeat domain. To be consistent with the conventional naming, we still refer the APC-ARM portion as the seven armadillo repeats Arm1 through Arm7 as previously identified $[1,3]$, and we regard the first four $\alpha$-helices of APC-PreARM as constituting armadillo repeats Arm-1 and Arm0. The length and pattern of the last $\alpha$-helix of APC-PreARM deviate much away from those of regular $\alpha$-helices of armadillo repeats, and thus we refer it as the insertion helix (H-Ins). Hydrophobic residues on helices Arm0-H2 and H-Ins of APC-PreARM pack extensively with those on helices Arm1-H3, Arm2-H1, and Arm2-H2 from APC-ARM, with a few hydrogen-bonding interactions at the periphery of the interface (Figure 2F).

\section{The interaction interface between APC and Asef}

Asef-ABR fits into a deep surface groove of APC-PreARM-ARM, which is formed by the $\mathrm{H} 3$ helices of Arm 1 through Arm4, as well as the H1 helix of Arm3 (Figure 1). The binding between APC and Asef results in the burial of $1,300 \AA^{2}$ exposed surface area for the structure of the APC-PreARM-ARM/Asef-ABR-SH3 complex. Because the resolution is higher for the APC-ARM/Asef-ABR complex structure $(2.3 \AA)$ than for the APC-PreARMARM/Asef-ABR-SH3 complex structure (3.0 $\AA$ ), we will mainly describe the interaction interface between APC and Asef in the structure of the APC-ARM/Asef-ABR complex.

At the interface between APC and Asef in the APCARM/Asef-ABR complex, there is an extensive hydrogen bond network. At the center of Asef-ABR, the carboxylate group of Asef-Glu183 accepts a chargestabilized hydrogen bond from the amino group of APCLys516, and forms a pair of hydrogen bonds with the main chain of APC-Gly511. The backbone of AsefGln184 makes two hydrogen bonds with the side chain of APC-Asn550, and the main chain of Asef-Ala186 forms a hydrogen bond with the side chain of APC-Asn507. At the N-terminal end of Asef-ABR, both Asef-Gly181 and Asef-Gly182 hydrogen bond to APC-Asn594. AsefGly181 also makes a hydrogen bond to APC-Arg549. At the C-terminal end of Asef-ABR, Asef-Glu189 forms a couple of hydrogen bonds with APC-Ser546 and also accepts a hydrogen bond from APC-Asn542. APC-Asn542 in turn forms a hydrogen bond with Asef-Asn188 (Figures 3A, 3B, and 4A).

In addition to the hydrogen-bonding network, van der Waals contacts are also important in stabilizing the interactions between APC and Asef. At the C-terminal part of Asef-ABR, Asef-Leu185, Asef-Ala186, Asef-Ile187, and Asef-Leu190 cluster around and make extensive van der Waals interactions with APC-Phe458. Asef-Leu185 is also surrounded by APC-Phe510 and the aliphatic portion of APC-Asn507. Moreover, the side chain of AsefAla186 fits snugly into a hydrophobic pocket on the surface of APC-ARM, which is created by APC-Phe458, APC-Met503, and the aliphatic portion of APC-Thr506. At the N-terminal part of Asef-ABR, both Asef-Gly180 and Asef-Gly181 contact APC-Trp593, and both AsefGly180 and Asef-Gly182 pack with APC-Trp553. The aliphatic portion of Asef-Glu183 is sandwiched between APC-Phe510 and APC-Trp553 (Figures 3A, 3B, and 4A).

Compared to the APC-ARM/Asef-ABR complex structure, some additional interactions are seen between APC and Asef in the structure of the APC-PreARMARM/Asef-ABR-SH3 complex. At the N-terminalextended region of Asef-ABR, Asef-Ser176 forms hydrogen bonds with both APC-Glu633 and APC-Thr675. Asef-Gly179 makes a hydrogen bond with APC-Trp593, and Asef-Pro178 makes van der Waals interaction with the aliphatic portion of APC-Thr675 (Figure 4B). At the C-terminal $\alpha$-helix of Asef-ABR, Asef-Ser192 makes a hydrogen bond with APC-Gln542 (Figure 4C).

Conservation of the Asef-binding groove on the APCPreARM-ARM surface

To see which part of the surface of APC-PreARMARM is most conserved, we aligned protein sequences of various APC homologs including human, Drosophila, and $C$. elegans APC, and mapped the conservation scores calculated using the ConSurf server (http://consurf.tau.ac.il) [30] to the surface of APC-PreARM-ARM structure. The binding groove for Asef-ABR on APCPreARM-ARM shows a striking conservation, while the rest of APC-PreARM-ARM surface does not present any comparable conservation (Figure 4D). This high conservation of Asef-binding groove on APC suggests that the interaction between APC and Asef is conserved throughout evolution. Although we did not find any Asef homolog in invertebrates, the high conservation of APCPreARM-ARM (Supplementary information, Figure S2) and the striking conservation of Asef-binding site on the APC-PreARM-ARM surface (Figure 4D) indicate that there might be homologs of Asef in invertebrates such as flies and worms, and that the recognition and activation 


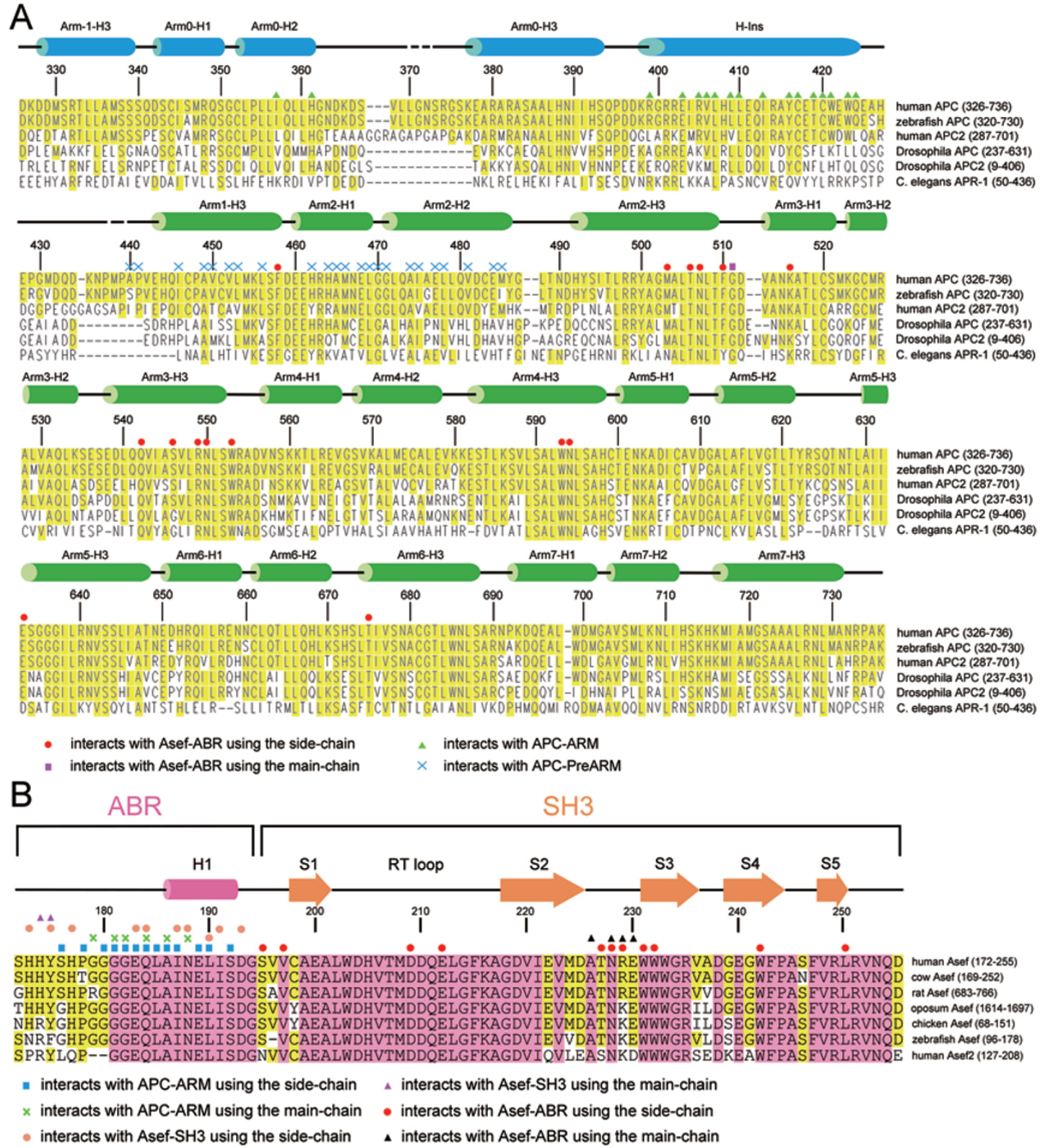

Figure 3 Sequence alignment and secondary structure elements of human APC (residues 326-736) and human Asef (residues 172-255). (A) Sequence alignment and secondary structure elements of human APC (residues 326-736). Human APC (residues 326-736), zebrafish APC (residues 320-730), human APC2 (residues 287-701), Drosophila APC (residues 237-631), Drosophila APC2 (residues 9-406), and C. elegans APR-1 (residues 50-436) protein sequences were aligned by the ClustalW method. Identical residues are highlighted in yellow. APC-ARM residues that interact with Asef-ABR using side chains or main chains are labeled by red circles or magenta squares, respectively. APC-PreARM and APC-ARM residues mediating interactions with each other are marked by green triangles and cyan crosses, respectively. Dashed lines indicate residues that are disordered in the crystal structure. (B) Sequence alignment and secondary structure elements of human Asef (residues 172255). Human Asef (RefSeq entry: NP_056135, residues 172-255), cow Asef (RefSeq entry: XP_582211, residues 169-252), rat Asef (RefSeq entry: XP_001055650, residues 683-766), opossum Asef (RefSeq entry: XP_001376703, residues 16141697), chicken Asef (RefSeq entry: XP_422582, residues 68-151), zebrafish Asef (RefSeq entry: XP_002660786, residues 96-178), and human Asef2 (RefSeq entry: NP_694568, residues 127-208) protein sequences were aligned by the ClustalW method. Residues identical in all seven Asef homologs are highlighted in pink, while other identical residues are highlighted in yellow. Asef-ABR residues that interact with APC-ARM using side chains or main chains are indicated by blue squares or green crosses, respectively. Asef-ABR residues that mediate interactions with Asef-SH3 using side chains or main chains are marked by orange circles or magenta triangles, respectively. Asef-SH3 residues that bind to Asef-ABR using side chains or main chains are designated by red circles or black triangles, respectively. 


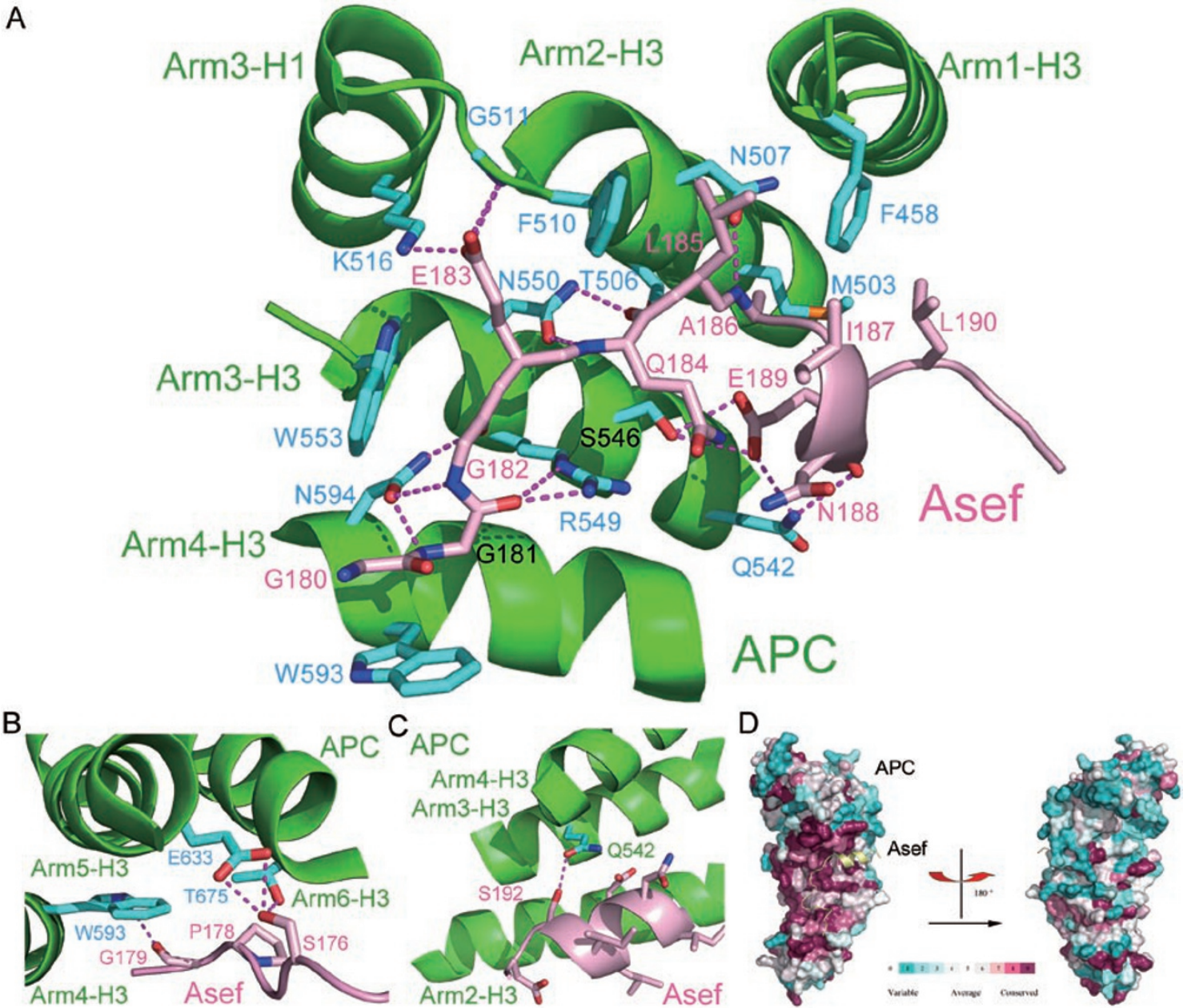

Figure 4 The interaction interface between APC and Asef. (A) Close-up view of the interface between APC and Asef in the $2.3 \AA$ resolution APC-ARM/Asef-ABR structure. APC is shown in green, with its side chains in cyan. Asef is shown in pink. Hydrogen bonds are represented by magenta dashed lines. Oxygen and nitrogen atoms are shown as red and blue balls, respectively. (B, C) Additional interactions between APC and the N-terminal end (B) or the C-terminal end (C) of Asef-ABR seen in the $3.0 \AA$ resolution APC-PreARM-ARM/Asef-ABR-SH3 structure. (D) The Asef-ABR-binding groove on APC-PreARM-ARM is highly conserved. APC-PreARM-ARM is shown as a surface representation and colored according to conservation scores. Asef-ABR is shown as a cartoon representation and colored in yellow.

of Asef might be a conserved function of APC.

Binding assays of the interaction between APC and Asef using site-directed mutants

To confirm our structural observations, we created single-point mutations in both APC and Asef, and examined the effects of these point mutations on the interaction between APC and Asef by the non-denaturing gel electrophoretic mobility shift assay (EMSA) and the isothermal titration calorimetry (ITC) assay.

Wild-type (WT) APC-PreARM-ARM could interact with WT Asef-ABR-SH3, as shown by the EMSA assay (Supplementary information, Figure S4A, note the decrease in the intensity of Asef band with increasing amount of APC). In addition, the dissociation constant $\left(K_{\mathrm{d}}\right)$ between WT APC-PreARM-ARM and WT AsefABR-SH3 proteins was measured to be $17.8 \mathrm{nM}$ by the ITC assay (Figure 5A, Table 2), indicating a tight binding between APC and Asef. On the other hand, consistent with their important roles in binding to Asef-ABR, point mutations of APC residues Asn507 (Supplementary information, Figures S4A and S5A, Table 2) or Asn550 (Figure 5B, Table 2, Supplementary information, Figure S4B) to Lys disrupted the interaction with Asef, with non-detectable $K_{\mathrm{d}}$ values from the ITC assay. APCAsn507 and APC-Asn550 form hydrogen bonds with 

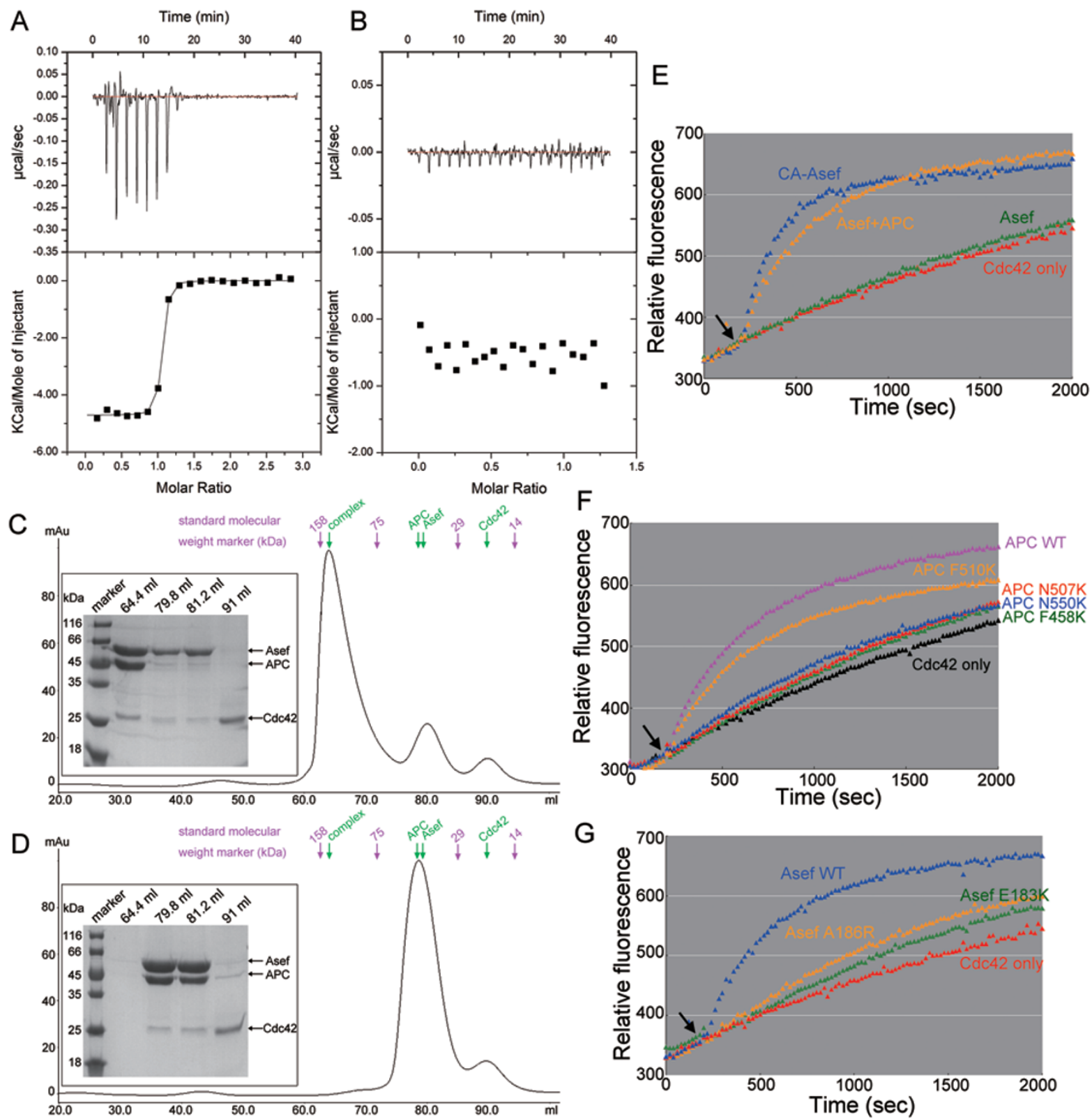

Figure 5 Point mutations of key interaction residues in APC and Asef disrupted the recognition and activation of Asef by APC. (A, B) Using the ITC assay to analyze the interaction between APC and Asef. (A) WT Asef-ABR-SH3 protein was titrated into the WT APC-PreARM-ARM protein, and the dissociation constant $\left(K_{d}\right)$ of the APC-PreARM-ARM/Asef-ABR-SH3 complex was measured to be $17.8 \mathrm{nM}$ (Table 2). (B) WT Asef-ABR-SH3 was titrated into the APC-PreARM-ARM N550K point mutant, and no detectable interaction was observed. (C, D) Using the HiLoad 16/60 Superdex 200 gel filtration chromatography assay to examine the APC/Asef/Cdc42 ternary complex formation. (C) WT APC-PreARM-ARM, WT Asef (170-632), and Cdc42 proteins could form a stable ternary complex. Elution volumes of standard molecular weight marker (shown in magenta), as well as elution volumes of APC-PreARM-ARM, WT Asef (170-632), and Cdc42 proteins by themselves (shown in green) were indicated above the chromatogram. Inset: samples from fractions of gel filtration chromatography were analyzed by SDSPAGE and Coomassie blue staining, with the elution volumes being indicated. (D) Point mutation N550K in APC-PreARMARM disrupted the APC/Asef/Cdc42 ternary complex formation. (E-G) Using the fluorescence-based guanine nucleotide exchange factor (GEF) activity assay to analyze the stimulation of Asef's GEF activity for Cdc42 by APC. (E) Asef (170-632) by itself had no detectable GEF activity for Cdc42, whereas the addition of APC-PreARM-ARM stimulated its GEF activity. Asef (277-632, CA-Asef) was constitutively active in the absence of APC. (F) Point mutations F510K, N550K, N507K, or F458K in APC-PreARM-ARM prevented the activation of the GEF activity of WT Asef (170-632). (G) GEF activities of Asef (170-632) point mutants E183K and A186R could not be stimulated by WT APC-PreARM-ARM. Black arrows indicate the time of APC/ Asef addition. 
Table 2 Dissociation constants $\left(K_{\mathrm{d}}\right)$ of the interaction between APC and Asef proteins

\begin{tabular}{llllll}
\hline Asef & APC & Asef/APC ratio & $K_{\mathrm{d}}(\mathrm{nM})$ & $\Delta H(\mathrm{kcal} / \mathrm{mol})$ & $T \Delta S(\mathrm{kcal} / \mathrm{mol})$ \\
\hline $170-271, \mathrm{WT}$ & $303-739, \mathrm{WT}$ & $0.998 \pm 0.004$ & $17.8 \pm 3.8$ & $-4.71 \pm 0.04$ & 5.87 \\
$170-271, \mathrm{WT}$ & $303-739, \mathrm{~N} 550 \mathrm{~K}$ & $\mathrm{n} / \mathrm{a}$ & $\mathrm{n} / \mathrm{a}$ & $\mathrm{n} / \mathrm{a}$ & $\mathrm{n} / \mathrm{a}$ \\
$170-271, \mathrm{WT}$ & $303-739, \mathrm{~N} 507 \mathrm{~K}$ & $\mathrm{n} / \mathrm{a}$ & $\mathrm{n} / \mathrm{a}$ & $\mathrm{n} / \mathrm{a}$ & $\mathrm{n} / \mathrm{a}$ \\
$170-271, \mathrm{E} 183 \mathrm{~K}$ & $303-739, \mathrm{WT}$ & $\mathrm{n} / \mathrm{a}$ & $\mathrm{n} / \mathrm{a}$ & $\mathrm{n} / \mathrm{a}$ & $\mathrm{n} / \mathrm{a}$ \\
$170-271, \mathrm{~A} 186 \mathrm{R}$ & $303-739, \mathrm{WT}$ & $\mathrm{n} / \mathrm{a}$ & $\mathrm{n} / \mathrm{a}$ & $\mathrm{n} / \mathrm{a}$ & $\mathrm{n} / \mathrm{a}$ \\
\hline
\end{tabular}

ITC experiments were performed at $25^{\circ} \mathrm{C}$. Typically, a $270-\mu \mathrm{M}$ Asef protein solution was injected into a $20-\mu \mathrm{M}$ APC protein solution. " $\mathrm{n} /$ a" refers to that no detectable interaction was observed. A negative value for $\Delta H$ would indicate an exothermic reaction while a positive value for $\Delta H$ would indicate an endothermic reaction.

Asef-Ala186 and Asef-Asn184, respectively, which are in the center of the core APC-binding motif of Asef-ABR [22]. APC-Asn507 also makes van der Waals contacts with Asef-Leu185 (Figure 4A). Thus APC-Asn507 and APC-Asn550 play crucial roles in the recognition of Asef by APC. Interestingly, it is reported that mutation of APC-Asn507 to Lys prevented full-length APC from binding Asef in vivo [12,22].

We also examined how point mutations in Asef would affect the APC/Asef complex formation. Our results showed that mutation of Asef-Glu183 to Lys (Supplementary information, Figures S4C and S5B) or mutation of Asef-Ala186 to Arg (Supplementary information, Figures S4D and S5C) greatly decreased the interaction between APC and Asef, with non-detectable $K_{\mathrm{d}}$ values (Table 2). Both Asef-Glu183 and Asef-Ala186 use a combination of hydrogen bonds and van der Waals interactions to make multiple contacts with APC. AsefGlu183 hydrogen bonds with APC-Lys516 and APCGly511, and uses its aliphatic side chain to form van der Waals contacts with APC-Phe510 and APC-Trp553. Asef-Ala186 fits nicely into a small hydrophobic pocket on APC-PreARM-ARM, formed by APC-Met503, APCPhe458, and APC-Thr506, and also uses its backbone to make a hydrogen bond with APC-Asn507 (Figure 4A). Therefore, mutations of these two residues would create a severe penalty for the APC/Asef interaction.

Activity assays of the stimulation of the guanine nucleotide-exchange factor activity of Asef by APC

On binding to APC, Asef is activated and becomes capable of recruiting the small GTPase Cdc42. Indeed, when we mixed purified WT APC-PreARM-ARM protein $(\sim 48 \mathrm{kDa})$, WT Asef (residues $170-632)$ protein $(\sim 51$ $\mathrm{kDa})$, and WT full-length Cdc42 protein $(\sim 21 \mathrm{kDa})$, and analyzed this mixture by gel filtration chromatography, we observed that APC, Asef, and Cdc42 formed a stable ternary protein complex, eluting as a $\sim 120 \mathrm{kDa}$ protein complex (Figure 5C). In contrast, point mutations of
N550K (Figure 5D) or N507K in APC (Supplementary information, Figure S6A), or point mutations of E183K (Supplementary information, Figure S6B) or A186R in Asef (Supplementary information, Figure S6C) resulted in dissociation of the APC/Asef/Cdc42 ternary complex.

On activation, Asef catalyzes the exchange of GDP for GTP in Cdc42. Indeed, when we performed the fluorescence-based GEF assay, the GEF activity of Asef (170632) was greatly stimulated in the presence of APCPreARM-ARM (Figure 5E). On the other hand, Asef (170-632) had no detectable GEF activity in the absence of APC, as compared with Cdc42 alone. In contrast, Asef (residues 277-632, CA-Asef), with deletion of the ABR and SH3 domains, possessed constitutively active GEF activity (Figure 5E). In addition, we examined how point mutations of APC-Asn550, APC-Asn507, APC-Phe458, and APC-Phe510, which play critical roles in binding to Asef, to Lys would affect APC's enhancement of the GEF activity of Asef. We found that all these mutations led to decreased APC-stimulated GEF activity of Asef to various extents (Figure 5F). Furthermore, Asef point mutants Asef-E183K and Asef-A186R could not be stimulated by WT APC-PreARM-ARM to activate Cdc42 (Figure $5 \mathrm{G}$ ). Therefore, our results of EMSA and ITC assays of APC/Asef interaction, together with our results of the APC/Asef/Cdc42 ternary complex formation assay and the fluorescence-based GEF activity assay, firmly supported our structural observations of key residues in APC and Asef important for the recognition and activation of Asef by APC.

\section{Conformational change of Asef-ABR on binding to $A P C$}

The structure of the SH3 domain of Asef in the APCPreARM-ARM/Asef-ABR-SH3 complex is almost the same as that in the Asef structure by itself $(0.371$ $\AA$ RMSD for aligned $\mathrm{C} \alpha$ atoms) $[22,24]$. On the other hand, binding to APC induced a dramatic conformational change in Asef-ABR (Figure 6A). Its C-terminal helix 
tilts $\sim 30^{\circ}$, with its packing with hydrophobic residues of Asef-SH3 still maintained. More importantly, the $\mathrm{N}$-terminal-extended region of Asef-ABR rotates $180^{\circ}$ and forms a second interface with the $\mathrm{SH} 3$ domain of Asef. At the center of this new interface, Asef-Tyr175 hydrogen bonds to Asef-Asp209, Asef-Glu212, and also makes van der Waals contacts with Asef-Trp231 and Asef-Trp242 from the SH3 domain. Interestingly, AsefTyr175 has been reported to be a phosphorylation site by Src family tyrosine kinases, and could be involved in the regulation of the activity of Asef [31]. In addition to Asef-Tyr175, Asef-His173, Asef-His174, and Asef-
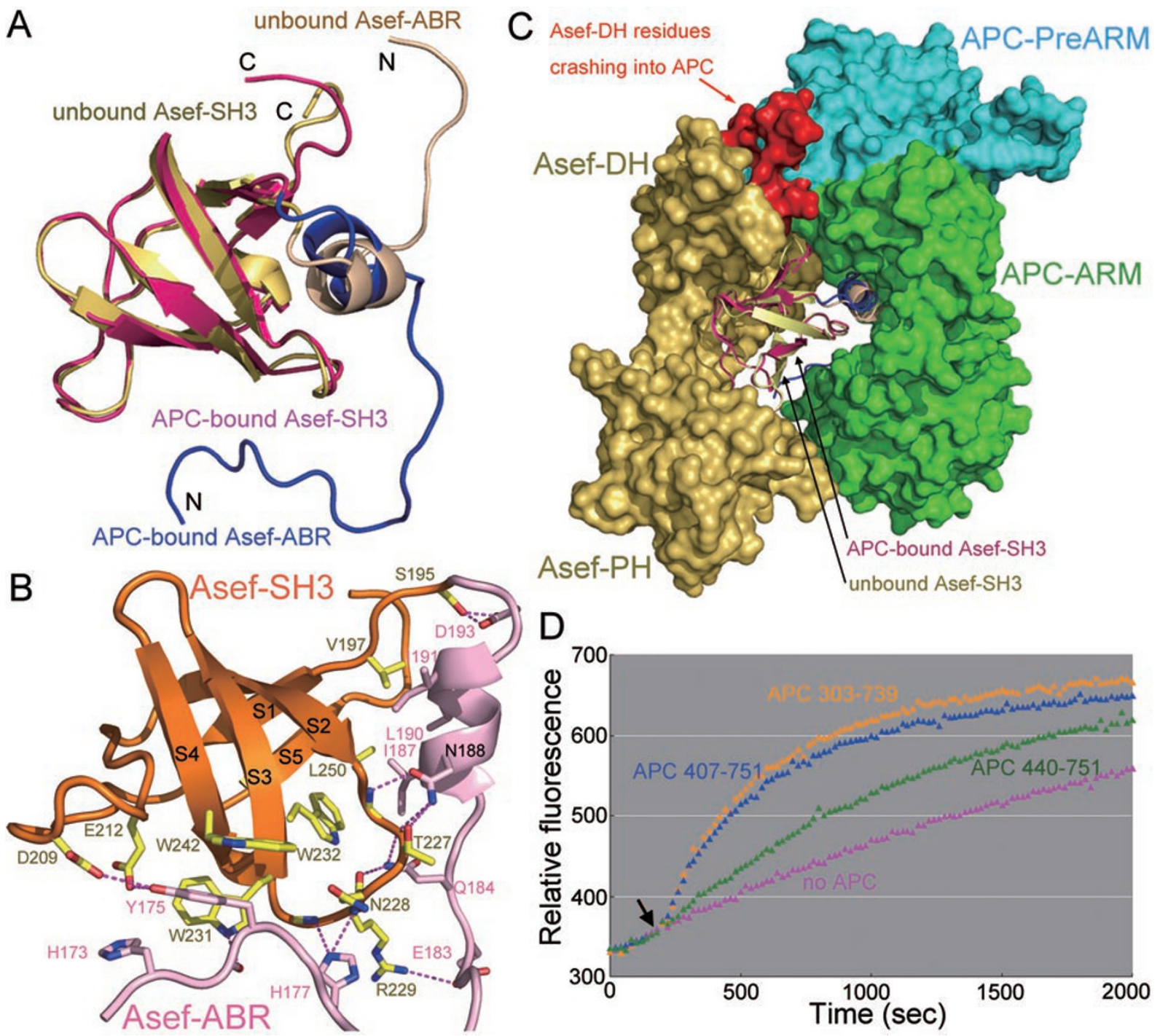

Figure 6 APC/Asef binding causes a conformational change of Asef-ABR, and would result in a steric clash between APC and Asef-DH domain. (A) Conformational change in Asef-ABR on binding to APC-PreARM-ARM. The structure of unbound Asef-SH3 (colored in yellow) [22], (PDB code 2PZ1) is superimposed onto that of APC-bound Asef-SH3 (colored in magenta). Unbound and APC-bound Asef-ABR are colored in wheat and blue, respectively. (B) Close-up view of the interface between Asef-ABR and Asef-SH3. Asef-ABR is shown in pink, and Asef-SH3 is shown in orange, with its side chains in yellow. Secondary structure elements of Asef-SH3 are marked. (C) APC binding to Asef would lead to a steric clash between APC and the Asef-DH domain. Unbound and APC-bound Asef-SH3 structures are superimposed. APC-ARM, APC-PreARM, and Asef$\mathrm{DH}-\mathrm{PH}$ are colored in green, cyan, and yellow, respectively. Asef-DH residues clashing with APC are highlighted in red. (D) Deletion of APC-PreARM was correlated with the decrease of APC's ability to stimulate the GEF activity of Asef for Cdc42. APC (303-739), APC (407-751), and APC (440-751), with different lengths of deletions of PreARM, were examined for their abilities to enhance the GEF activity of Asef. Black arrow indicates the time of APC/Asef addition. 
His177 from the ABR region are also involved in this new interface with the SH3 domain (Figure 6B).

Binding between APC and Asef might result in a steric clash between APC and the DH domain of Asef

In the Asef structure by itself, the SH3 domain interacts with the $\mathrm{DH}$ and $\mathrm{PH}$ domains, and occupies the Cdc42-binding site on the $\mathrm{DH}$ domain, so that the entry of Cdc42 is blocked. Therefore, the GEF activity of Asef is autoinhibited by this intra-molecular interaction between the $\mathrm{SH} 3$ domain and the $\mathrm{DH}$ and $\mathrm{PH}$ domains [22, 24]. The SH3 domain has to dissociate from the $\mathrm{DH}$ and $\mathrm{PH}$ domains in order for $\mathrm{Cdc} 42$ to be recognized by the DH domain. APC binding to Asef results in the release of autoinhibition of Asef. However, it is not clear how the activation of Asef by APC is accomplished. Superimposition of the structure of Asef-SH3 domain in the APC-PreARM-ARM/Asef-ABR-SH3 complex and that in unbound Asef [22] showed that on binding to APC, the Asef-DH domain would be in collision with APCPreARM and the N-terminal part of APC-ARM (Figure 6C). Therefore, upon APC/Asef binding, there has to be a conformational change in Asef or/and APC to avoid this steric clash. This conformational change presumably initiates the separation between Asef-DH and Asef-SH3 domains, thus $\mathrm{Cdc} 42$ could be recruited by the Asef-DH domain, and thus can be activated [22].

In accordance with this hypothesis, in which APC-PreARM is supposed to play a major role in the activation of Asef, we found that APC (303-739), which possesses a complete PreARM portion, caused the highest activation of the GEF activity of Asef. On the other hand, APC (407-751), which has partial deletion of the PreARM portion, could not stimulate the GEF activity of Asef as potently as APC (303-739). Furthermore, APC (440-751), in which the PreARM portion is completely deleted, had even weaker ability to enhance the GEF activity of Asef (Figure 6D). Therefore, the PreARM portion is required for full activation of Asef by APC, and the deletion of APC-PreARM is correlated with the decrease of activation of Asef by APC.

\section{Discussion}

Recognition between APC and Asef results in the relief of autoinhibition of Asef and the recruitment of small GTPase Cdc42. Our crystal structures of the APC/ Asef complex provide a structural clue for this important process. Docking of the structure of autoinhibited Asef to the structure of the APC-PreARM-ARM/Asef-ABRSH3 complex showed that the Asef-DH domain would collide with APC-PreARM-ARM upon recognition of
Asef by APC. To avoid this steric clash, the Asef-DH and Asef-SH3 domains have to be separated, thus relieving the inhibition of the Asef-DH domain by the Asef-SH3 domain (Figure 6C). In support of this hypothesis, we found that the association between Asef and APC is very strong, with the dissociation constant $K_{\mathrm{d}}$ measured to be $17.8 \mathrm{nM}$ by the ITC assay (Figure 5A, Table 2). Unfortunately, we were not able to measure the dissociation constant between the Asef-DH and Asef-SH3 domains, as it is an intra-molecular interaction in nature. Nonetheless, we found that the inter-molecular interaction between separated Asef-DH and Asef-SH3 domains was very weak based on our GST pull-down binding results (data not shown). Therefore, we suggest that the strength of interaction between Asef-DH and Asef-SH3 domains is not comparable with that between Asef and APC, and that the binding of APC with Asef could provide enough driving force to initiate the separation between the $\mathrm{DH}$ and $\mathrm{SH} 3$ domains of Asef.

Interestingly, when we performed the normal mode analysis on Asef using the autoinhibited Asef structure [22] as the initial model, we found that Asef had a basic vibrational mode where its $\mathrm{DH}$ and $\mathrm{PH}$ domains moved towards each other and extended out from the autoinhibited state (Supplementary information, Figure S7). The normal mode analysis of APC-ARM also suggested that APC-ARM had a basic vibrational mode where the N-terminal part of APC-ARM (and thus APC-PreARM together with it) wagged side-to-side relative to the Cterminal part of APC-ARM (Supplementary information, Figure S8A). In addition, superimpositions of structures of APC-ARM by itself, APC-ARM in complex with Asef-ABR, and APC-PreARM-ARM in complex with Asef-ABR-SH3 also revealed that APC-ARM has an intrinsic flexibility between its $\mathrm{N}$ - and $\mathrm{C}$-terminal parts (Supplementary information, Figure S8B), in accordance with the suggested intrinsic flexibility for $\beta$-cateninARM domain [28]. Therefore, it is possible that the breathing modes of APC-PreARM-ARM and Asef allow APC to access and bind to an "open" (i.e., uninhibited) form of Asef, which exists transiently from the breathing/thermal fluctuations. Once the "open" form of Asef is bound by APC, the steric clash of Asef-DH with APCPreARM and the N-terminal part of APC-ARM would prevent Asef-DH and Asef-SH3 from re-associating, therefore Asef is prevented from returning to the "closed" (i.e., autoinhibited) form. Furthermore, the strong interaction between APC-ARM and Asef-ABR substantially stabilizes the "open" form of Asef, and thus shifts the equilibrium of Asef from the "closed" form to the "open" form. In this way, the autoinhibition of Asef is relieved. Consistent with this hypothesis, our fluorescence-based 
GEF assay revealed that the PreARM portion of APC was indeed crucial for the activation of Asef, and that its deletion was correlated with the impairment of APC's stimulation of the GEF activity of Asef (Figure 6D).

Asef was originally identified as a GEF specific for the small GTPase Rac1 [11]. However, subsequent studies showed that the specificities of Asef and its close homolog Asef2 were exclusively for Cdc42 [18-19, 22]. We have performed gel filtration chromatography assays to examine whether APC could induce the interaction between Asef and Rac1, as well as that between Asef and Cdc42. We were not able to observe the APC-induced binding between Asef and Rac1 (data not shown), whereas we could consistently observe the APC-stimulated interaction between Asef and Cdc42 (Figure 5C). Therefore, we limited our investigation and discussion only on how APC stimulates the GEF activity of Asef for Cdc42, and did not pursue further on whether and how APC would activate the GEF activity of Asef for Rac1.

In colorectal cancers, APC is usually present in a truncated form, with an intact PreARM-ARM domain. Asef is constitutively activated by truncated APC, stimulates the GEF activity of $\mathrm{Cdc} 42$, and promotes tumor invasion and angiogenesis [17, 20-22, 25-27]. It has been noted that compounds targeting Asef could be novel anti-tumor reagents [26, 27]. Our crystal structures of the APC/Asef complex show that Asef-ABR binds to a deep surface groove of APC-PreARM-ARM, which is lined with both hydrophobic and charged residues and is suitable for interaction with small molecules. Therefore, our structures not only reveal how APC recognizes and activates Asef, but also provide a potential anti-tumor drug target. Small molecules capable of disrupting APC binding and activation of Asef could become interesting lead compounds against colorectal cancer.

\section{Materials and Methods}

\section{Protein expression and site-directed mutagenesis}

APC (residues 407-751), APC (residues 303-739), APC (residues 440-751), Asef (residues 170-271), Asef (residues 170-632), Asef (residues 277-632), and full-length Cdc42 were cloned into a pET28a- (Novagen) derived vector, and overexpressed as Histagged proteins. All proteins were overexpressed in E. coli strain BL21 (DE3). Point mutations were introduced by the overlapping PCR method, and identities of individual clones were verified by sequencing.

\section{Protein purification}

Cells were harvested and resuspended in the $\mathrm{Ni}^{2+}$ columnbinding buffer $(25 \mathrm{mM}$ Tris- $\mathrm{HCl}, \mathrm{pH} 8.0,300 \mathrm{mM} \mathrm{NaCl}$, and 20 $\mathrm{mM}$ imidazole). After sonication, cell lysates were centrifuged and purified by the $\mathrm{Ni}^{2+}$ affinity chromatography (GE Healthcare).
APC (407-751) After the $\mathrm{Ni}^{2+}$ affinity chromatography, His-APC (407-751) was purified by the Superdex 200 gel filtration chromatography (GE Healthcare) equilibrated with buffer containing 20 $\mathrm{mM}$ Tris- $\mathrm{HCl}, \mathrm{pH} 8.0,300 \mathrm{mM} \mathrm{NaCl}, 0.5 \mathrm{mM}$ EDTA, and $2 \mathrm{mM}$ DTT. Peak fractions were combined and concentrated to $18 \mathrm{mg} /$ $\mathrm{ml}$.

Selenomethionine-substituted APC (407-751) Selenomethionine (SeMet)-substituted APC (407-751) was expressed using the methionine-autotrophic E. coli strain B834 (DE3) cultured in M9 media, and purified by the same procedure as that for the native protein, except that $5 \mathrm{mM}$ DTT was used in the gel filtration buffer.

Complex of APC (407-751) and Asef (170-194) The Asef (170194) peptide was synthesized chemically and purified by the reverse-phase HPLC. The complex was prepared by mixing purified His-APC (407-751) protein with Asef (170-194) peptide with a 1:1 molar ratio.

Complex of APC (303-739) and Asef (170-271) APC (303-739) and Asef (170-271) proteins were purified by the $\mathrm{Ni}^{2+}$ affinity chromatography, and mixed with a 1:2 molar ratio to make sure that APC (303-739) was fully complexed with Asef (170-271). The protein complex was further purified by the Superdex 200 gel filtration chromatography, with equilibration buffer containing 10 $\mathrm{mM}$ Tris- $\mathrm{HCl}, \mathrm{pH} 7.0,250 \mathrm{mM} \mathrm{NaCl}$, and $2 \mathrm{mM}$ DTT. The eluted APC (303-739)/Asef (170-271) protein complex was at a 1:1 molar ratio, and extra Asef (170-271) protein was eluted as a second peak. Peak fractions of APC (303-739)/Asef (170-271) complex were combined and concentrated to $20 \mathrm{mg} / \mathrm{ml}$.

Cdc42 His-tagged full-length $\mathrm{Cdc} 42$ was purified by the $\mathrm{Ni}^{2+}$ affinity chromatography and was further purified by the Superdex 200 gel filtration chromatography, with equilibration buffer containing 20 mM Tris- $\mathrm{HCl}, \mathrm{pH}$ 8.0, 150 mM NaCl, 1 mM DTT, $10 \mu \mathrm{M}$ GDP, and $1 \mathrm{mM} \mathrm{MgCl}_{2}$.

Crystallization, data collection, and structure determination SeMet-substituted APC (407-751) Native and SeMet-substituted APC (407-751) protein crystals were grown at $14{ }^{\circ} \mathrm{C}$ by the sitting-drop vapor-diffusion method, with the reservoir solution containing $0.1 \mathrm{M}$ Tris-HCl, $\mathrm{pH}$ 8.0, 25\% PEG-3350, and $180 \mathrm{mM}$ ammonium sulfate. Both crystals grew as clusters of thin plates. Fortunately, one SAD data set of Se-APC (407-751) at $1.6 \AA$ was collected at beamline BL17U1 at Shanghai Synchrotron Radiation Facility (China). The crystal was in space group P1 and contained two molecules in each asymmetric unit. Initial SAD phases were determined using the Autosol module of PHENIX [32], followed by density modification and automatic model building using the AutoBuild module of PHENIX. The remaining model was built manually with COOT [33]. The refinement was performed by the CCP4i [34] program REFMAC. The final model has an $R / R_{\text {free }}$ factor of $16.8 \% / 20.1 \%$, and includes residues $407-428$ and $434-$ 738 of APC. Residues 429-433 and 739-751 had poor electron density in the map, and therefore presumed to be disordered. In the Ramachandran plot, $98.5 \%$ and $1.5 \%$ of residues are in the most favored and allowed regions, respectively. The model quality was checked with the CCP4i program PROCHECK. 
APC (407-751)/Asef (170-194) complex Crystals of the APC (407-751)/Asef (170-194) complex were grown at $14{ }^{\circ} \mathrm{C}$ by the hanging-drop vapor-diffusion method. The reservoir solution contained 0.1 M Bis-Tris-propane, $\mathrm{pH}$ 9.0, 25\% PEG-1500, and 0.1 M $\mathrm{NaCl}$. Crystals belonged to space group $\mathrm{C} 2$, with three complexes in each asymmetric unit. Data sets were collected at beamline BL17U1 at Shanghai Synchrotron Radiation Facility (China). The structure was solved by the molecular replacement method with the CCP4i program PHASER using the APC (407-751) structure as the searching model. Model building was performed by COOT. Due to strong anisotropy, reflection files were truncated at the diffraction anisotropy server to improve the electron density. After refinement by REFMAC, the model has an $R / R_{\text {free }}$ factor of $20.8 \% / 25.7 \%$. The final model includes residues $407-427$ and $436-$ 736 of APC, and residues 180-192 of Asef. Residues 428-435 and 737-751 of APC, as well as residues 170-179 and 193-194 of Asef were not visible in the electron density map. In the Ramachandran plot, $98.7 \%$ and $1.3 \%$ of residues are in the most favored and allowed regions, respectively. The model quality was checked with PROCHECK.

APC (303-739)/Asef (170-271) complex Crystals of the APC (303-739)/Asef (170-271) complex were grown at $4{ }^{\circ} \mathrm{C}$, using the hanging-drop vapor-diffusion method. The reservoir solution contained $0.1 \mathrm{M}$ sodium citrate, $\mathrm{pH}$ 5.6, $1 \mathrm{M}$ lithium sulfate, and $0.5 \mathrm{M}$ ammonium sulfate. Crystals belonged to the R3 space group, with two complexes in each asymmetric unit. Data sets were collected at the BL17A beamline at Photon Factory, KEK (Japan). The structure was solved using the molecular replacement method with the CCP4i program PHASER, using the structure of APC (407751) and the structure of Asef by itself (PDB code 2PZ1) [22] as searching models. Reflection files were truncated at the diffraction anisotropy server to improve the electron density. The model was built by COOT and then refined by REFMAC. The final model has an $R / R_{\text {free }}$ factor of $25.1 \% / 28.6 \%$, and includes residues 326 $369,373-437$, and 440-736 of APC and residues 171-255 of Asef. Electron density was not interpretable for residues 303-325, 370$372,438-439$, and 737-739 of APC, as well as residues 170 and 256-271 of Asef, and therefore could not be modeled. In the Ramachandran plot, $96.4 \%, 3.3 \%$, and $0.3 \%$ of residues are in the most favored, allowed, and disallowed regions, respectively. The model quality was checked with PROCHECK.

\section{Molecular graphics}

All protein structure figures were generated with PyMOL (http:// pymol.sourceforge.net). Sequence conservation of Asef-ABR projected onto the protein surface of APC-PreARM-ARM was generated by the ConSurf server (http://consurf.tau.ac.il) [30].

Binding assay between APC and Asef using the non-denaturing gel electrophoretic mobility shift assay

Purified WT or mutant APC (303-739) protein was mixed with WT or mutant Asef (170-271) protein on ice for $20 \mathrm{~min}$. The protein mixture was then analyzed by non-denaturing gel electrophoretic mobility shift assay using the Tris-Borate gel running buffer, $\mathrm{pH} 8.0$, followed by Coomassie Blue staining.

\section{Isothermal titration calorimetry assay}

Isothermal titration calorimetry experiments were performed using an ITC200 system (MicroCal) at $25^{\circ} \mathrm{C}$. The buffer contained $50 \mathrm{mM}$ HEPES, pH 7.5, $300 \mathrm{mM} \mathrm{NaCl}$, and $1 \mathrm{mM}$ EDTA. Proteins were centrifuged and degassed before the experiment. Typically, a $270-\mu \mathrm{M}$ WT or point mutant Asef (170-271) protein solution was injected 20 times in $2 \mu \mathrm{l}$ aliquots into a $300-\mu 1$ sample-cell containing WT or point mutant APC (303-739) protein at a concentration of $20 \mu \mathrm{M}$. Data were fit with a nonlinear leastsquare routine using a single-site binding model with Origin for ITC version 7.0 (MicroCal), varying the stoichiometry (n), the enthalpy of the reaction $(\Delta H)$, and the association constant $\left(K_{\mathrm{a}}\right)$.

\section{The APC/Asef/Cdc42 ternary protein complex formation as- say using gel filtration chromatography}

Purified WT or point mutant APC (303-739) protein, WT or point mutant Asef (170-632) protein, and WT full-length Cdc42 protein were mixed on ice for $20 \mathrm{~min}$. The protein mixture was then loaded onto a HiLoad 16/60 Superdex 200 gel filtration chromatography column. The buffer contained $20 \mathrm{mM}$ Tris-HCl, $\mathrm{pH} 8.0$, $300 \mathrm{mM} \mathrm{NaCl}, 14 \mathrm{mM}$ beta-mercaptoethanol, and $1 \mathrm{mM}$ EDTA. Samples from selected Superdex 200 fractions were analyzed by SDS-PAGE and Coomassie Blue staining.

\section{The fluorescence-based GEF activity assay}

N-methylanthraniloyl (mant)-GTP incorporation into Cdc42 was carried out with a Perkin-Elmer LS-50B spectrometer at $25^{\circ} \mathrm{C}$. Exchange reaction assay mixtures contained $20 \mathrm{mM}$ Tris- $\mathrm{HCl}, \mathrm{pH} 7.5$, $100 \mathrm{mM} \mathrm{NaCl}, 5 \mathrm{mM} \mathrm{MgCl}, 1 \mathrm{mM}$ DTT, 5\% (v/v) glycerol, 400 $\mathrm{nM}$ mant-GTP (Sigma), and $2 \mu \mathrm{M}$ Cdc42, and were pre-equilibrated by continuous stirring. After equilibration, $1 \mu \mathrm{M}$ Asef or $1 \mu \mathrm{M}$ Asef plus $1 \mu \mathrm{M}$ APC was added to the reaction mixture, and the relative fluorescence was monitored. The excitation wavelength was $360 \mathrm{~nm}$, and the emission wavelength was $440 \mathrm{~nm}$. For experiments including APC, Asef and APC proteins were incubated on ice for $20 \mathrm{~min}$ before being added to the GEF reactions.

\section{Normal mode analysis}

The structures of Asef (PDB code 2PZ1) and APC (residues 407-751) were used for a series of computational simulations. Normal mode analysis was conducted using the web server developed by Delarue et al. (http://lorentz.immstr.pasteur.fr/nomad-ref.php). The cutoff radius Rc was set to be $10 \AA$. Files of the $7^{\text {th }}$ normal mode (i.e., the $1^{\text {st }}$ vibrational mode) generated by the server were used for analysis.

\section{Accession codes}

The atomic coordinates and structure factors of APC (407-751), the APC (407-751)/Asef (170-194) complex, and the APC (303739)/Asef (170-271) complex have been deposited in the Protein Data Bank with accession numbers 3NMW, 3NMX, and 3NMZ, respectively.

\section{Acknowledgments}

We thank Dr Ning Zheng (University of Washington, USA) for critical reading of our manuscript, Dr Xi He (Children's Hospital Boston, USA) and Dr Tetsu Akiyama (University of Tokyo, Japan) for the generous gifts of human APC and Asef cDNA, respectively. We thank Dr Jiahai Zhou (Chinese Academy of Sciences), Dr Mingjin Fang (Fudan University, China), Dr Zhiyong Lou (Tsinghua 
University, China), and Dr Yanhui Xu (Fudan University, China) for assistance in crystal diffraction data reduction and structure determination. We thank the staffs at the beamline BL17U1 at Shanghai Synchrotron Radiation Facility (China) and beamlines BL5 and BL17A at KEK Photon Factory (Japan) for assistance in data collection. This work was supported by grants from the National Natural Science Foundation of China (30900225, 30821005 and 90919021), Innovation Program of Shanghai Municipal Education Commission (09ZZ23), the "Dawn" Program of Shanghai Education Commission (08SG11), Shanghai Pujiang Program (09PJ1405500), the Key Project of Chinese Ministry of Education (109060), the Program for Professor of Special Appointment (Eastern Scholar) at Shanghai Institutions of Higher Learning, the National Comprehensive Technology Platforms for Innovative Drug R\&D (2009ZX09301-007), the State Key Laboratory of Bioorganic and Natural Products Chemistry, Shanghai Institute of Organic Chemistry (China), and the State Key Laboratory of Drug Research (China).

\section{References}

1 Kinzler KW, Vogelstein B. Lessons from hereditary colorectal cancer. Cell 1996; 87:159-170.

2 Rustgi AK. The genetics of hereditary colon cancer. Genes Dev 2007; 21:2525-2538.

3 Hirschl D, Bayer P, Muller O. Secondary structure of an armadillo single repeat from the APC protein. FEBS Lett 1996; 383:31-36.

4 Graham TA, Weaver C, Mao F, Kimelman D, Xu W. Crystal structure of a beta-catenin/Tcf complex. Cell 2000; 103:885896.

5 Ha NC, Tonozuka T, Stamos JL, Choi HJ, Weis WI. Mechanism of phosphorylation-dependent binding of APC to betacatenin and its role in beta-catenin degradation. Mol Cell 2004; 15:511-521.

6 Huber AH, Weis WI. The structure of the beta-catenin/Ecadherin complex and the molecular basis of diverse ligand recognition by beta-catenin. Cell 2001; 105:391-402.

7 Sampietro J, Dahlberg CL, Cho US, Hinds TR, Kimelman D, $\mathrm{Xu}$ W. Crystal structure of a beta-catenin/Bc19/Tcf4 complex. Mol Cell 2006; 24:293-300.

8 Xing Y, Clements WK, Kimelman D, Xu W. Crystal structure of a beta-catenin/axin complex suggests a mechanism for the beta-catenin destruction complex. Genes Dev 2003; 17:27532764.

9 Xing Y, Clements WK, Le Trong I, et al. Crystal structure of a beta-catenin/APC complex reveals a critical role for APC phosphorylation in APC function. Mol Cell 2004; 15:523-533.

$10 \mathrm{Xu} \mathrm{W}$, Kimelman D. Mechanistic insights from structural studies of beta-catenin and its binding partners. J Cell Sci 2007; 120:3337-3344.

11 Kawasaki Y, Senda T, Ishidate T, et al. Asef, a link between the tumor suppressor APC and G-protein signaling. Science 2000; 289:1194-1197.

12 Watanabe T, Wang S, Noritake J, et al. Interaction with IQGAP1 links APC to Rac1, Cdc42, and actin filaments during cell polarization and migration. Dev Cell 2004; 7:871-883.

13 Jimbo T, Kawasaki Y, Koyama R, et al. Identification of a link between the tumor suppressor APC and the kinesin superfam- ily. Nat Cell Biol 2002; 4:323-327.

14 Aoki K, Taketo MM. Adenomatous polyposis coli (APC): a multi-functional tumor suppressor gene. J Cell Sci 2007; 120:3327-3335.

15 MacDonald BT, Tamai K, He X. Wnt/beta-catenin signaling: components, mechanisms, and diseases. Dev Cell 2009; 17:926.

16 Takacs CM, Baird JR, Hughes EG, et al. Dual positive and negative regulation of wingless signaling by Adenomatous Polyposis Coli. Science 2008; 319:333-336.

17 Akiyama T, Kawasaki Y. Wnt signaling and the actin cytoskeleton. Oncogene 2006; 25:7538-7544.

18 Gotthardt K, Ahmadian MR. Asef is a Cdc42-specific guanine nucleotide exchange factor. Biol Chem 2007; 388:67-71.

19 Hamann MJ, Lubking CM, Luchini DN, Billadeau DD. Asef2 functions as a Cdc42 exchange factor and is stimulated by the release of an autoinhibitory module from a concealed C-terminal activation element. Mol Cell Biol 2007; 27:1380-1393.

20 Kawasaki Y, Sato R, Akiyama T. Mutated APC and Asef are involved in the migration of colorectal tumor cells. Nat Cell Biol 2003; 5:211-2115.

21 Kawasaki Y, Sagara M, Shibata Y, Shirouzu M, Yokoyama S, Akiyama T. Identification and characterization of Asef2, a guanine-nucleotide exchange factor specific for Rac1 and Cdc42. Oncogene 2007; 26:7260-7267.

22 Mitin N, Betts L, Yohe ME, Der CJ, Sondek J, Rossman KL. Release of autoinhibition of ASEF by APC leads to CDC42 activation and tumor suppression. Nat Struct Mol Biol 2007; 14:814-823.

23 Thiesen S, Kubart S, Ropers HH, Nothwang HG. Isolation of two novel human RhoGEFs, ARHGEF3 and ARHGEF4, in 3p13-21 and 2q22. Biochem Biophys Res Commun 2000; 273:364-369.

24 Murayama K, Shirouzu M, Kawasaki Y, et al. Crystal structure of the Rac activator, Asef, reveals its autoinhibitory mechanism. J Biol Chem 2007; 282:4238-4242.

25 Kawasaki Y, Tsuji S, Sagara M, Echizen K, Shibata Y, Akiyama T. Adenomatous polyposis coli and Asef function downstream of hepatocyte growth factor and phosphatidylinositol 3-kinase. J Biol Chem 2009; 284:22436-22443.

26 Kawasaki Y, Tsuji S, Muroya K, et al. The adenomatous polyposis coli-associated exchange factors Asef and Asef2 are required for adenoma formation in $A P C^{\mathrm{Min} /+}$ mice. $E M B O R E P$ 2009; 10:1355-1362.

27 Kawasaki Y, Jigami T, Furukawa S, et al. The adenomatous polyposis coli-associated guanine nucleotide exchange factor Asef is involved in angiogenesis. J Biol Chem 2010; 285:1199-1207.

28 Huber AH, Nelson WJ, Weis WI. Three-dimensional structure of the armadillo repeat region of beta-catenin. Cell 1997; 90:871-872.

29 Choi H, Weis WI. Structure of the armadillo repeat domain of Plakophilin 1. J Mol Biol 2005; 346:367-376.

30 Landau M, Mayrose I, Rosenberg Y, et al. ConSurf 2005: The projection of evolutionary conservation scores of residues on protein structures. Nucleic Acids Res 2005; 33:W299-W302.

31 Itoh RE, Kiyokawa E, Aoki K, Nishioka T, Akiyama T, Matsuda M. Phosphorylation and activation of the Rac1 and Cdc42 GEF Asef in A431 cells stimulated by EGF. J Cell Sci 
2008; 121:2635-2642.

32 Adams PD, Grosse-Kunstleve RW, Hung LW, et al. PHENIX: Building new software for automated crystallographic structure determination. Acta Crystallogr D Biol Crystallogr 2002; 58:1948-1954.
33 Emsley P, Cowtan K. Coot: Model-building tools for molecular graphics. Acta Crystallogr D 2004; 60:2126-2132.

34 Collaborative Computational Project, Number 4. The CCP4 suite: programs for protein crystallography. Acta Crystallogr D Biol Crystallogr 1994; 50:760-763.

(Supplementary information is linked to the online version of the paper on the Cell Research website.) 